\title{
Pleiotrophin regulates microglia-mediated neuroinflammation
}

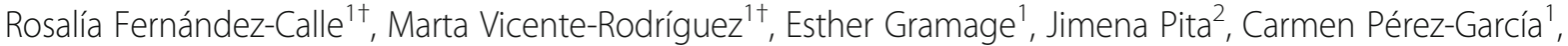 \\ Marcel Ferrer-Alcón ${ }^{3}$, María Uribarri' ${ }^{3}$ María P. Ramos ${ }^{2}$ and Gonzalo Herradón ${ }^{1 *}$
}

\begin{abstract}
Background: Pleiotrophin (PTN) is a cytokine found highly upregulated in the brain in different disorders characterized by overt neuroinflammation such as neurodegenerative diseases, drug addiction, traumatic injury, and ischemia. In the present work, we have explored whether PTN modulates neuroinflammation and if Toll-like receptor 4 (TLR4), crucial in the initiation of an immune response, is involved.

Methods: In immunohistochemistry assays, we studied lipopolysaccharide (LPS, $7.5 \mathrm{mg} / \mathrm{kg}$ i.p.)-induced changes in glial fibrillary acidic protein (GFAP, astrocyte marker) and ionized calcium-binding adaptor molecule 1 (Iba1, microglia marker) expression in the prefrontal cortex (PFC) and striatum of mice with transgenic PTN overexpression in the brain (PTN-Tg) and in wild-type (WT) mice. Cytokine protein levels were assessed in the PFC by X-MAP technology. The influence of TLR4 signaling in LPS effects in both genotypes was assessed by pretreatment with the TLR4 antagonist (TAK-242, $3.0 \mathrm{mg} / \mathrm{kg}$ i.p.). Murine BV2 microglial cells were treated with PTN $(0.5 \mu \mathrm{g} / \mathrm{ml})$ and LPS $(1.0 \mu \mathrm{g} / \mathrm{ml})$ and assessed for the release of nitric oxide (NO).

Results: We found that LPS-induced microglial activation is significantly increased in the PFC of PTN-Tg mice compared to that of WT mice. The levels of TNF-a, IL-6, and MCP-1 in response to LPS were significantly increased in the PFC of PTN-Tg mice compared to that of WT mice. Pretreatment with TAK-242 efficiently blocked increases in cytokine contents in a similar manner in both genotypes. Concomitant incubation of BV2 cells with LPS and PTN significantly potentiated the production of NO compared to cells only treated with LPS.

Conclusions: Our findings identify for the first time that PTN is a novel and potent regulator of neuroinflammation. Pleiotrophin potentiates LPS-stimulated microglia activation. Our results suggest that regulation of the PTN signaling pathways may constitute new therapeutic opportunities particularly in those neurological disorders characterized by increased PTN cerebral levels and neuroinflammation.
\end{abstract}

Keywords: Microgliosis, Microglia activation, Midkine, Neuroimmune response, Neuroinflammation, Pleiotrophin, TLR4

\section{Background}

Inflammation is a key event in the healing process of the damaged tissue, and activation of the innate immune system is fundamental in the response to inflammation. When prolonged, however, inflammation can become deleterious. Within the central nervous system (CNS), the two main players in neuroinflammation are glial cells: microglia, the resident macrophages in the CNS,

\footnotetext{
* Correspondence: herradon@ceu.es

${ }^{\dagger}$ Equal contributors

'Pharmacology Lab, Department of Pharmaceutical and Health Sciences, Facultad de Farmacia, Universidad CEU San Pablo, Urb. Montepríncipe, 28668 Boadilla del Monte, Madrid, Spain

Full list of author information is available at the end of the article
}

and astrocytes [1]. Toll-like receptors (TLRs), which are expressed in the rodent microglia and astrocytes [2], are key molecules in the activation of innate immunity during CNS damage. Activation of TLRs triggers the downstream stimulation of nuclear factor- $\mathrm{kB}(\mathrm{NF \kappa} B)$ and the induction of genes that encode inflammation-associated molecules and cytokines including TNF- $\alpha$, IL-1 $1 \beta$, IL-6, iNOS, and COX2 [2-4].

Ever-growing evidence points to a key role of inflammatory processes in a broad spectrum of diseases including traumatic brain injury, chronic neurodegenerative diseases, neuropathic pain, ischemia, and neuropsychiatric disorders including drug addiction [5-7]. 
Psychostimulants such as amphetamine and its derivatives cause neuroinflammation and limit neurogenesis and induce blood-brain barrier (BBB) damage $[8,9]$. All these effects induced by amphetamines are important for the dopaminergic injury induced by these drugs in the nigrostriatal pathway [10], which is the same circuitry affected in Parkinson's disease (PD). Interestingly, recent evidence indicate a 3-fold increased risk of PD in these drug addicts [11], suggesting that common pathogenic mechanisms could underlie both diseases, PD and amphetamines abuse, one of which could be neuroinflammation $[9,12]$. In the search for validation of new biomarkers and for the development of new drugs that could modulate the inflammatory processes underlying these and other diseases of the CNS [13], our strategy was to identify proteins with known regulatory functions in inflammation, whose levels of expression are upregulated after amphetamine administrations and in the neurodegenerative areas of the brain of PD patients.

Pleiotrophin (PTN) is a cytokine that is upregulated in different brain areas after administration of different drugs of abuse including amphetamine [14, 15] and in the nigrostriatal pathway of patients with PD [16]. PTN is important for CNS repair and for survival and differentiation of dopaminergic neurons [6]. In addition, evidence points to a modulatory role of PTN on inflammation. In peripheral organs, PTN is known to induce inflammatory mediators [17] and its expression levels are significantly reduced by administration of anti-inflammatory drugs [18]. Little is known about a possible role of PTN on neuroinflammation. Transgenic mice with PTN overexpression in the brain (PTN-Tg) show a 4-fold increased amphetamineinduced striatal astrogliosis compared to wild-type (WT) mice [19]. However, PTN genetically deficient (PTN-/-) mice show a modest $\sim 20 \%$ increase in amphetamineinduced astrocytosis, possibly reflecting proinflammatory compensatory mechanisms [19-21]. We hypothesize that PTN is a novel modulator of neuroinflammation. To test this hypothesis, we have now comparatively studied the astrocytic response, microglial activation, and cytokine release induced by lipopolysaccharide (LPS) in PTN-Tg and in WT mice. Since previous studies suggest a possible contribution of PTN to Toll-like receptor 4 (TLR4)-mediated immune response [22] and TLR4 plays a pivotal role in neuroinflammation, we have also investigated the possible differential contribution of TLR4 to the neuroinflammatory processes induced by LPS in both genotypes.

\section{Methods}

\section{Animals}

PTN-Tg mice on a C57BL/6J background were generated by pronuclear injection as previously described [23, 24]. The acceptor vector contained the regulatory regions responsible for tissue-specific expression of Thy-1 gene, which drives neuron-specific expression of transgenes $[25,26]$. PTN-specific overexpression in different brain areas, including an $\sim 3$-4-fold upregulation in the prefrontal cortex (PFC) and a $20 \%$ increase of PTN protein levels in striatum, was established by quantitative real-time polymerase chain reaction (qRT-PCR), in situ hybridization, and by western blot $[23,27,28]$.

We used male PTN-Tg and WT animals of 9-10 weeks (20-25 g). Mice were housed under controlled environmental conditions $\left(22 \pm 1{ }^{\circ} \mathrm{C}\right.$ and a 12-h light/12-h dark cycle) with free access to food and water.

All the animals used in this study were maintained in accordance with the European Union Laboratory Animal Care Rules (86/609/ECC directive), and the protocols were approved by the Animal Research Committee of USP-CEU.

\section{Treatments}

To test genotypic differences in LPS-induced neuroinflammation and the involvement of TLR4 in LPS effects in PTN-Tg and WT mice, we assessed the effects of LPS in mice pretreated with the TLR4 antagonist TAK-242 (Merck Millipore, Madrid, Spain). For this purpose, mice were injected (i.p.) with TAK-242 $(3 \mathrm{mg} / \mathrm{kg})$ or saline (10 ml/kg; control) 30 minutes before a single i.p. injection of LPS $(7.5 \mathrm{mg} / \mathrm{kg})$ or saline $(10 \mathrm{ml} / \mathrm{kg}$; control). As a result, we obtained four experimental groups in both genotypes: saline + saline (Sal-Sal), saline + LPS (Sal-LPS), TAK-242 + saline (TAK-Sal), and TAK-242 + LPS (TAK-LPS). For immunohistochemistry analysis, animals were sacrificed $16 \mathrm{~h}$ after LPS or last saline administration ( $n=4-5 /$ group/genotype) by perfusion with $4 \%$ p-formaldehyde. For tisular analysis of cytokine levels, animals were decapitated $16 \mathrm{~h}$ after LPS or the last saline administration ( $n=5 /$ group/genotype) and the PFC was rapidly removed and frozen in dry ice and stored to $-80{ }^{\circ} \mathrm{C}$ until the protein extraction procedure.

\section{Immunohistochemistry analysis}

Mice were transcardially perfused with $4 \%$ p-formaldehyde; the brains were removed and conserved in p-formaldehyde for $24 \mathrm{~h}$ and then transferred to a $30 \%$ sucrose solution containing $0.02 \%$ sodium azide for storage at $4{ }^{\circ} \mathrm{C} ; 30-\mu \mathrm{m}$ PFC and striatal free-floating sections were processed as previously described [20, 21, 29]. Immunohistochemistry studies were performed in one slice per $180 \mu \mathrm{m}$ (the PFC from the bregma $-3.08 \mathrm{~mm}$ to $-2.46 \mathrm{~mm}$; the striatum from the bregma $1.54 \mathrm{~mm}$ to $-0.10 \mathrm{~mm}$ ).

In order to study gliosis, sections were incubated overnight at $4{ }^{\circ} \mathrm{C}$ with anti-glial fibrillary acidic protein (GFAP; Millipore, Madrid, Spain; 1:1000) and antiionized calcium-binding adaptor molecule 1 (Iba1, Wako, Osaka, Japan; 1:1000) antibodies, following by 30min incubation with the Alexa-Fluor-555 and Alexa-Fluor- 
488 corresponding secondary antibody (Invitrogen, Waltham, MA, USA; 1:500). Sections were mounted on gelatin-coated slides and coverslipped with Fluoromount medium. Photomicrographs were captured with a digital camera coupled to an optical microscope (DM5500B, Leica, Solms, Germany). Analysis was performed using ImageJ (NIH, Bethesda, MD, Version 1.50f), in the three most central slices of each area. Iba1+ cells and GFAP+ astrocytes were counted in whole sections of the PFC and in $1100 \mu \mathrm{m} \times 1400 \mu \mathrm{m}$ standardized areas in the striatum as previously described [21, 29]. In the case of Iba1-ir, total marked area was calculated as overall image fluorescence, subtracting the mean background fluorescence. Computer-based analysis of the morphology of individual Iba1+ cells was performed using the "Analyze Particle" function in ImageJ software. Images were segmented and smoothed to best fit cell shape. To be sure to select only cells entirely present in the acquired field, cells with an area of $>25 \mu \mathrm{m}^{2}$ were analyzed. The objects meeting the minimum size to be analyzed were measured for the following parameters: area, perimeter, and circularity. The soma size (cell area) is expressed in square micrometers. The perimeter was calculated based on the outline length of a given object. It measures the length around the periphery of each soma and is expected to be higher in activated and hypertrophic cells. Circularity was calculated by the following formula: $4 \pi \times$ (area/perimeter $^{2}$ ). This parameter varies from 0 (linear polygon) to 1 (perfect circular object). Mean single-cell values for each parameter were used for statistics.

\section{Cytokine levels}

Approximately $5 \mathrm{mg}$ of each sample of the PFC $(n=5 /$ group) was homogenized in $100 \mu \mathrm{l}$ of homogenization buffer $(0.05 \%$ Tween 20 and protease inhibitor cocktail (Thermo Fisher Scientific Inc., Waltham, MA, USA) in PBS, pH 7.2). After 3 cycles of $1 \mathrm{~min}$ and $50 \mathrm{~Hz}$ in the tissuelyzer (Quiagen, Germantown, Maryland, USA), samples were centrifuged at $11000 \times g$ for $30 \mathrm{~min}$ at $4{ }^{\circ} \mathrm{C}$. After centrifugation, supernatants were transferred to a new tube and stored at $-80{ }^{\circ} \mathrm{C}$ until the assay. Total protein content of each sample was measured using the BCA protein assay kit (Thermo Fisher Scientific Inc., Waltham, MA, USA). Levels of tumor necrosis factor- $\alpha$ (TNF- $\alpha)$, interleukin $1 \beta$ (IL-1 $\beta$ ), interleukin 6 (IL-6), monocyte chemoattractant protein-1 (MCP-1), interleukin 4 (IL-4), and interleukin 10 (IL-10) were measured by X-Map technology using a Milliplex MADPK-71K adipokine kit according to the manufacturer's description (Merck Millipore, Spain).

\section{BV2 cell cultures}

\section{BV2 murine microglial cells}

BV2 murine microglial cells were a generous gift from Professor Antonio Cuadrado (Instituto de Investigaciones
Biomédicas "Alberto Sols" (IIBM), Madrid, Spain). Cells were routinely maintained in RPMI-1640 medium with fetal bovine serum (10\%), penicillin $(100 \mathrm{U} / \mathrm{ml})$, streptomycin $(100 \mu \mathrm{g} / \mathrm{ml})$, and L-glutamine $(4 \mathrm{mM})$ at $37{ }^{\circ} \mathrm{C}$ in $5 \% \mathrm{CO}_{2}$ humidified air following conditions used by others [30, 31]. Prior to each experiment, cells were grown for $24 \mathrm{~h}$ on 96 -well plates at a concentration of $1 \times 10^{4}$ cells per well.

\section{Measurement of NO production}

Nitric oxide (NO) production was quantified by nitrite accumulation in the culture medium using the Griess reactive (2.25\% sulfanilamide and $0.22 \% \mathrm{~N}$-(1-naphthyl)ethylenediamine dihydrochloride), according to protocols previously described [32, 33]. After fasting the cells for $24 \mathrm{~h}, \mathrm{BV} 2$ cells were stimulated with different concentrations of PTN $(0.05 \mu \mathrm{g} / \mathrm{ml}$ or $0.5 \mu \mathrm{g} / \mathrm{ml})$, with or without LPS $(1.0 \mu \mathrm{g} / \mathrm{ml})$, for another $24 \mathrm{~h}$. The concentrations of PTN were selected to correlate with the PTN overexpression in the brain of PTN-Tg mice and are within the range of concentrations used before to test the effects of PTN in neuronal injury [34] and in the expansion of human stem cells [35]. The NO production by cells was quantified in a microplate reader (Versa-Max, Molecular Devices, Sunnyvale, CA, USA) at $540 \mathrm{~nm}$ and then calculated with reference to the standard curve generated with $\mathrm{NaNO}_{2}$.

\section{Statistics}

Data are presented as mean \pm standard error of the mean (SEM). Data obtained from image analysis of the striatal and PFC immunostaining and cytokine levels were analyzed using two-way ANOVA considering genotype and treatment as variants. Relevant differences were analyzed by post hoc comparisons with Bonferroni's post hoc tests. Data obtained from BV2 cells were analyzed using one-way ANOVA followed by post hoc comparisons with Tukey's post hoc tests. $P<0.05$ was considered as statistically significant. All statistical analyses were performed using Graph-Pad Prism program (San Diego, CA, USA).

\section{Results}

Differential regulation of LPS-induced astrocytic and microglial responses in the PFC of PTN-Tg mice. Effect of TAK-242 on LPS-induced microglial response and changes in cytokine contents in the PFC of PTN-Tg and WT mice PTN is a cytokine highly upregulated in the brain in different CNS disorders characterized by neuroinflammation [6]. We aimed to study the modulatory role of PTN overexpression (3-fold) in the PFC of PTN-Tg mice [23, 24] on LPS-induced neuroinflammation. To investigate proinflammatory responses in these experiments, we tested the astrocytic and microglial response in PFC sections of WT 
and PTN-Tg mice treated with saline (Sal-Sal) or LPS (Sal-LPS). Astrocyte activation was assessed by morphology and expression of GFAP, an astrocyte-specific intermediate filament protein [36]. LPS treatment tended to increase the number of GFAP+ astrocytes in the PFC of WT mice, being these cells characterized by large densely stained cell bodies as well as long and extensive processes compared to saline-treated animals (Fig. 1). In contrast, these effects on GFAP+ IR induced by LPS were blocked in PTN-Tg mice (Fig. 1).

Previous studies have linked LPS-induced activation of microglia and production of proinflammatory factors to brain damage and neurodegeneration [37]. To investigate proinflammatory responses in this experiment, PFC sections were immunostained with anti-Iba1 microglial antibody. In the saline control groups, microglial cells have resting morphology (Fig. 2a, Sal-Sal). Immunohistochemistry for Iba1 did not reflect significant changes in the number of Iba1+ cells in the PFC of WT and PTN-Tg mice after LPS treatment (Fig. 2b). However, we observed a clearly

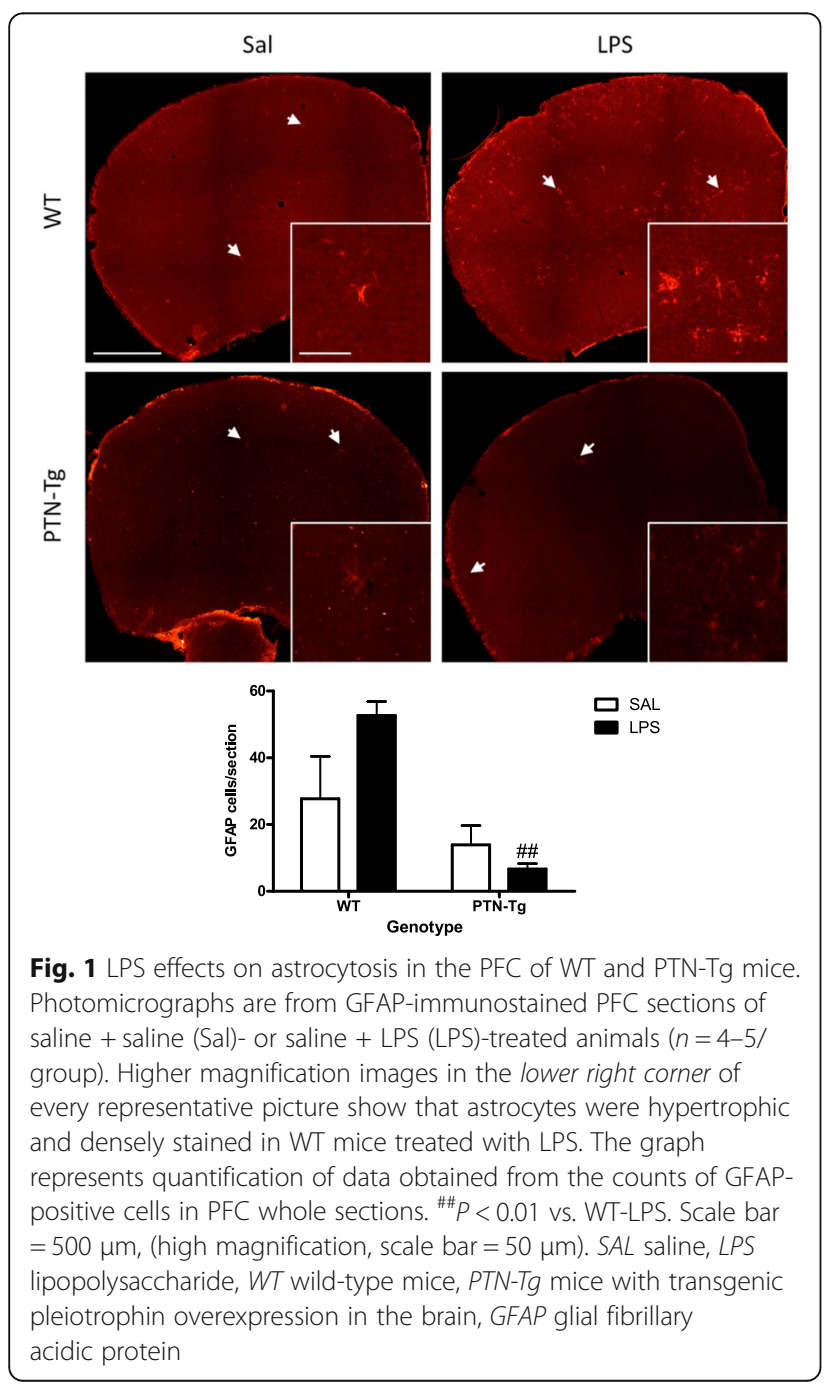

enhanced hypertrophism characterized by activation, soma enlargement, and sprouting of new ramifications in LPStreated PTN-Tg mice compared to that in LPS-treated WT mice and saline-treated mice from the same genotype (Fig. 2a, Sal-LPS). Accordingly, the total marked area (Fig. 2c) tended to increase in LPS-treated PTN-Tg mice compared to that in saline-treated mice. As expected, the Iba1+ cell area was increased in LPS-treated mice of both genotypes compared with that in saline-treated mice (Fig. 2d); however, LPS induced an increase in cell area that was significantly enhanced in PTN-Tg mice compared to that in WT mice (Fig. 2d). The increase in the perimeter was also higher in LPS-treated PTN-Tg mice (Fig. 2e). The decrease caused by LPS in the circularity index was more pronounced in PTN-Tg mice (Fig. 2f). Overall, the data demonstrate that LPS-induced microglial response is increased in the PFC of PTN-Tg mice compared to that of WT mice. Interestingly, we observed that the morphological changes induced by LPS in microglial cells of both genotypes were not prevented by the previous administration of the TLR4 antagonist TAK-242 (Fig. 2, TAK-LPS).

We measured the contents of different cytokines in the PFC of mice from both genotypes. Confirming the neuroimmune response to LPS administration, we found that the levels of proinflammatory cytokines such as TNF- $\alpha$, IL-6, and MCP-1 were many-fold upregulated in the PFC of LPS-treated WT mice compared to those of the saline-treated group (Fig. 3, Sal-LPS vs. Sal-Sal). More importantly, we found a highly significant $\sim 7$-fold upregulation of the levels of TNF- $\alpha$ in the PFC of LPStreated PTN-Tg mice compared to that of WT mice (Fig. 3). Similarly, the levels of IL-6 in the PFC of LPStreated PTN-Tg mice were increased compared to those of WT mice (Fig. 3). Interestingly, pretreatment with TAK-242 efficiently blocked LPS-induced increases of TNF- $\alpha$ and IL- 6 in the PFC of mice from both genotypes (Fig. 3, Sal-LPS vs. TAK-LPS). In addition, we found significantly higher levels of MCP-1 in the PFC of LPS-treated PTN-Tg mice compared to those of WT mice although, in this case, LPS effect on MCP-1 levels was partially blocked by previous administration of TAK-242 in both genotypes (Fig. 3). In contrast, we did not observe relevant genotypic differences in the levels of IL-1 $\beta$ (Fig. 3). Concerning the anti-inflammatory cytokines IL-10 and IL-4, we did not find genotype- or treatment-related significant differences in their contents in the PFC of LPS-treated PTN-Tg mice compared to those of WT mice (Fig. 3).

Overall, the data demonstrate that LPS-induced microglial activation is enhanced in the PFC of PTN-Tg mice compared to that of WT animals. Interestingly, the data suggest that TLR4 activation is crucial for LPSinduced cytokine release in both genotypes but does not play a significant role in LPS-induced microglial morphological response in either genotype. 

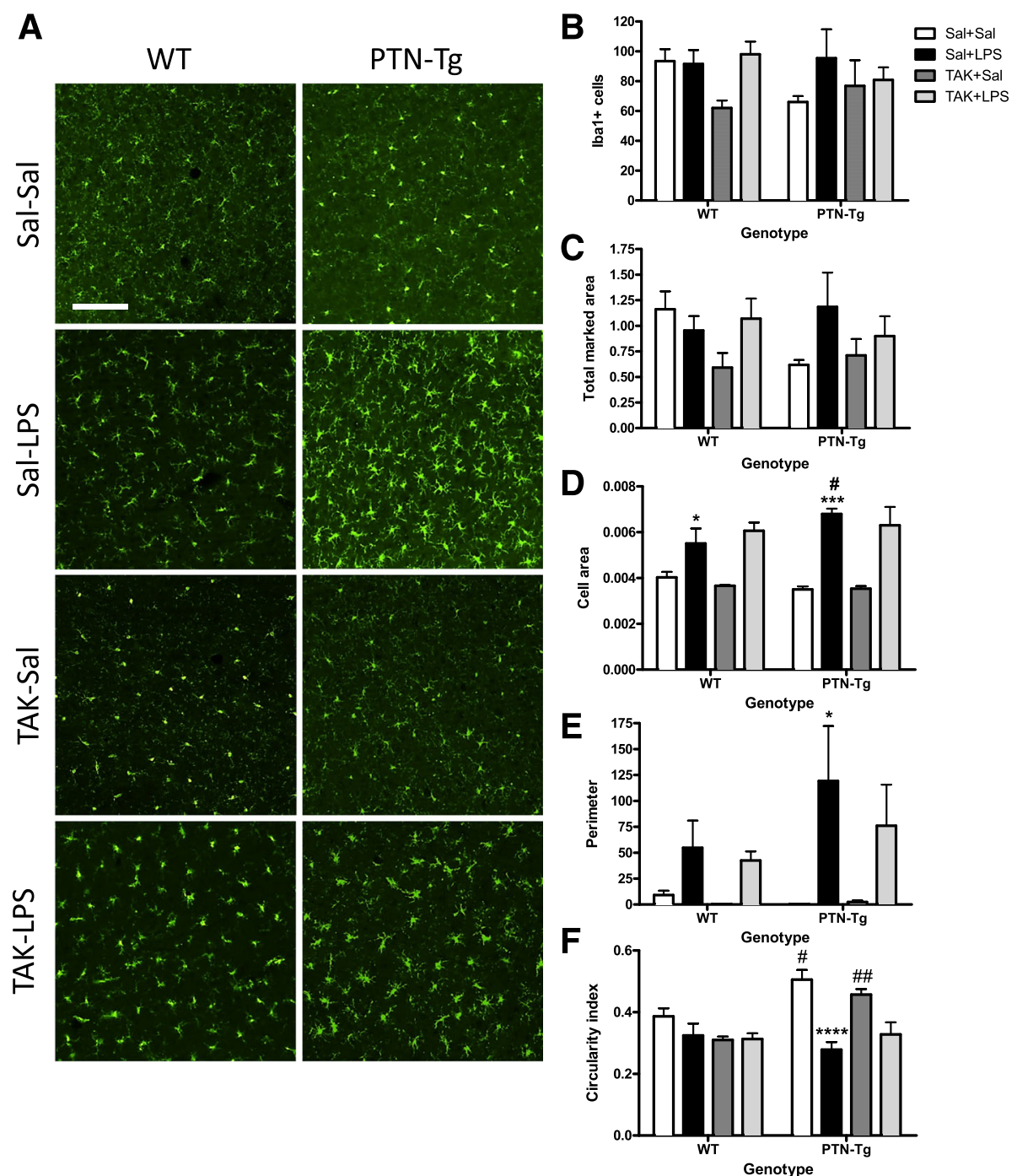

Fig. 2 Effects of TAK-242 and LPS on microglia activation in the PFC of PTN-Tg mice. Photomicrographs are from Iba-1-immunostained PFC sections of saline (Sal)- or TAK-242 (TAK)-pretreated and saline (Sal)- or LPS-treated animals ( $n=4-5 /$ group) (a). Graphs represent quantification of data (mean \pm SEM) obtained from the counts of Iba-1-positive cells $(\mathbf{b})$, total marked area $(\mathbf{c})$, cell area $(\mathbf{d})$, soma perimeter $(\mathbf{e})$, and circularity index $(\mathbf{f})$ in PFC whole sections. ${ }^{*} P<0.05,{ }^{* * *} P<0.001,{ }^{* * *} P<0.0001$ vs. Sal + Sal within the same genotype. ${ }^{\#} P<0.05,{ }^{\# \#} P<0.01$ vs. WT within the same treatment. Scale bar $=100 \mu \mathrm{m}$. SAL saline, LPS lipopolysaccharide, TAK TAK-242, WT wild-type mice, PTN-Tg mice with transgenic pleiotrophin overexpression in the brain, Ibal ionized calcium-binding adaptor molecule 1

\section{Effects of TAK-242 and LPS in the striatum of WT and \\ PTN-Tg mice}

To investigate the effects of a more modest PTN overexpression ( 20\%, PTN-Tg mouse striatum) [28] on LPSinduced neuroinflammation, we tested the astrocytic and microglial response in striatal sections of WT and PTN-Tg mice treated with saline (Sal-Sal) or LPS (Sal-LPS). We did not find genotypic- or treatment-related significant differences in the number of GFAP+ cells between groups (Fig. 4). Similarly to PFC, LPS treatment tended to increase the number of GFAP+ cells in the striatum of WT mice, whereas this tendency was absent in PTNTg mice (Fig. 4). Concerning microglia (Fig. 5), immunohistochemistry for Iba1 did not reflect significant changes in the number of Iba1+ cells or in the total marked area in the striatum of WT and PTN-Tg mice after LPS treatment (Fig. 5a, b, c). However, signs of hypertrophism, soma enlargement, and sprouting of new ramifications in LPS-treated animals were observed in both genotypes compared to those in saline-treated groups (Fig. 5a). The Iba1+ cell area was significantly increased in both genotypes but especially in LPS-treated PTN-Tg mice compared to that in WT mice (Fig. 5d). On the other hand, the increase in the perimeter was higher in LPS-treated WT mice (Fig. 5e), whereas no differences between groups were found in 

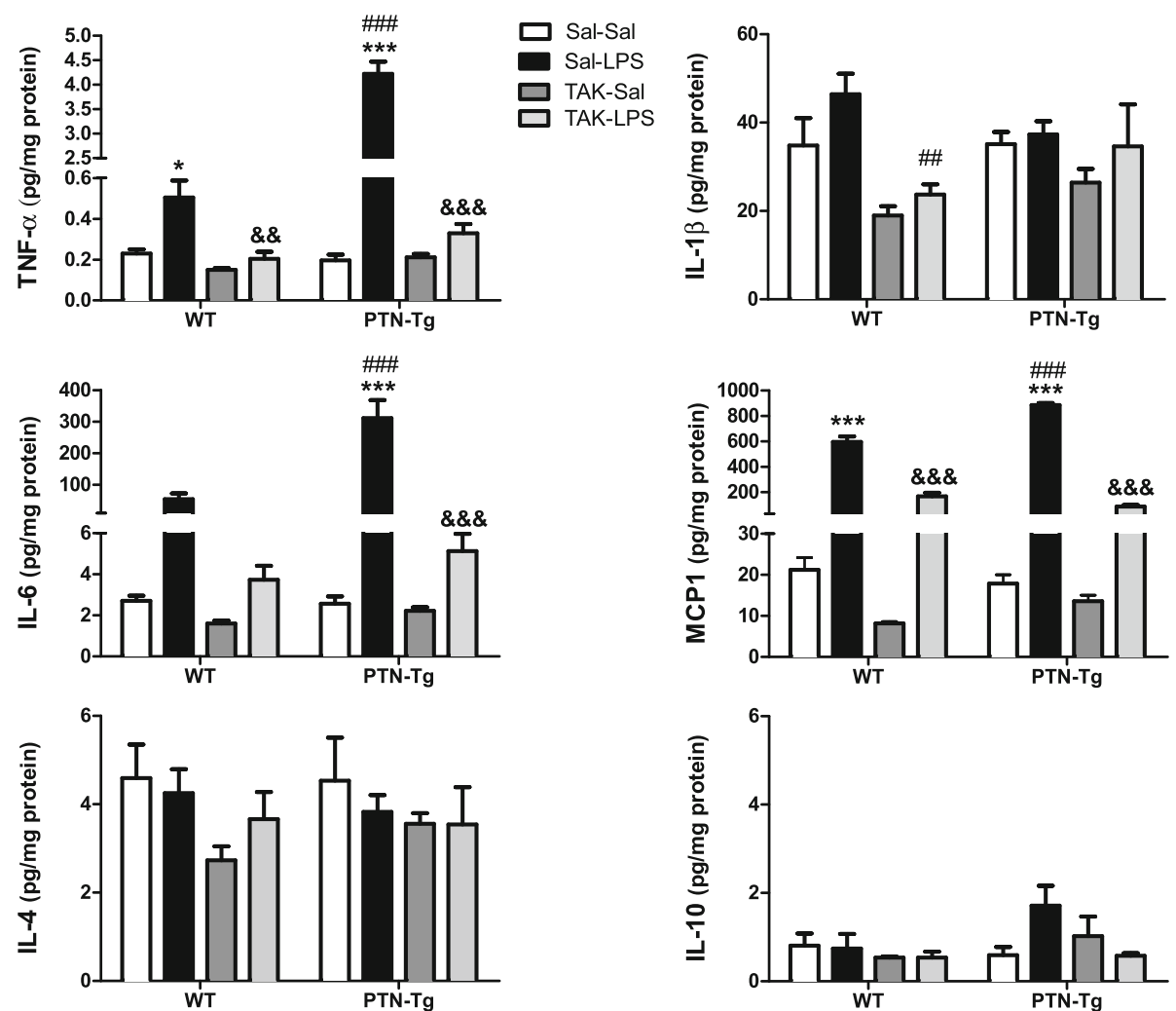

Fig. 3 Effects of TAK-242 and LPS on cytokine expression in the PFC of PTN-Tg mice. TNF-a, II-1 $\beta, I L-6, M C P-1$, IL-4, and IL-10 protein levels measured using a Milliplex system in PFC of mice pretreated with saline (Sal) or TAK-242 (TAK) and treated with saline (Sal) or LPS $(n=5 / \mathrm{group})$. ${ }^{*} P<0.05$, ${ }^{* * *} P<0.001$ vs. Sal + Sal within the same genotype. ${ }^{\# \#} P<0.01$, ${ }^{\# \# \#} P<0.001$ vs. WT within the same treatment. $\& \& P<0.01, \& \& \& P<0.001$ vs. Sal + LPS within the same genotype. SAL saline, LPS lipopolysaccharide, TAK TAK-242, WT wild-type mice, PTN-Tg mice with transgenic pleiotrophin overexpression in the brain, TNF Tumor necrosis factor-a, IL-1 $\beta$ interleukin $1 \beta$, IL-6 interleukin 6, MCP-1 monocyte chemoattractant protein-1, IL-4 interleukin 4, IL-10 interleukin 10

the case of the circularity index (Fig. 5f). We did not observe significant effects of pretreatment with TAK242 on LPS-induced effects on microglia (Fig. 5). Overall, the data demonstrate that LPS-induced microglial response in the striatum is more modest than that in the PFC and that the moderate PTN overexpression in this brain area of PTN-Tg mice does not play a significant modulatory role on LPS effects.

\section{Pleiotrophin potentiates NO production in LPS-stimulated BV2 cells}

To test the possible direct effects of PTN on murine microglia in vitro, we investigated its effects on the production of $\mathrm{NO}$, which is a key inflammatory mediator in LPS-stimulated BV2 microglia. First, we did not detect any effect in BV2 cells incubated with PTN alone $(0.05$ and $0.5 \mu \mathrm{g} / \mathrm{ml}$, Fig. 6). LPS $(1.0 \mu \mathrm{g} / \mathrm{ml})$ induced a significant increase in the production of NO compared with control cells (Fig. 6). Concomitant incubation of BV2 cells with LPS $(1.0 \mu \mathrm{g} / \mathrm{ml})$ and PTN $(0.5 \mu \mathrm{g} / \mathrm{ml})$ significantly potentiated the production of $\mathrm{NO}$ compared to cells only treated with LPS (Fig. 6).

\section{Discussion}

Pleiotrophin is a cytokine that is found highly upregulated in diverse pathologies of the CNS characterized by overt neuroinflammation including neurodegenerative diseases, addictive disorders, ischemia, and neuropathic pain $[6,38,39]$. The goal of this study was to investigate whether pleiotrophin regulates the astrocytic response and the activation of microglia in the brain after an inflammatory challenge. We used the systemic LPS treatment acute inflammation murine brain model. In the in vivo study, we found that LPS induced a moderate increase in the number of GFAP+ astrocytes in the PFC and striatum of WT mice. After LPS treatment, astrocytes appear hypertrophic, particularly in the PFC, suggesting a LPS-induced upregulation of GFAP protein concentrations in astrocytes of WT mice. This response was significantly reduced in the PFC of LPS-treated PTN-Tg mice, an area with a more significant PTN overexpression in this genotype [24]. We previously found a small $(\sim 20 \%)$ increase of striatal GFAP+ astrocytes in amphetamine-treated PTN knockout (PTN-/-) mice which could be attributed to compensatory mechanisms 


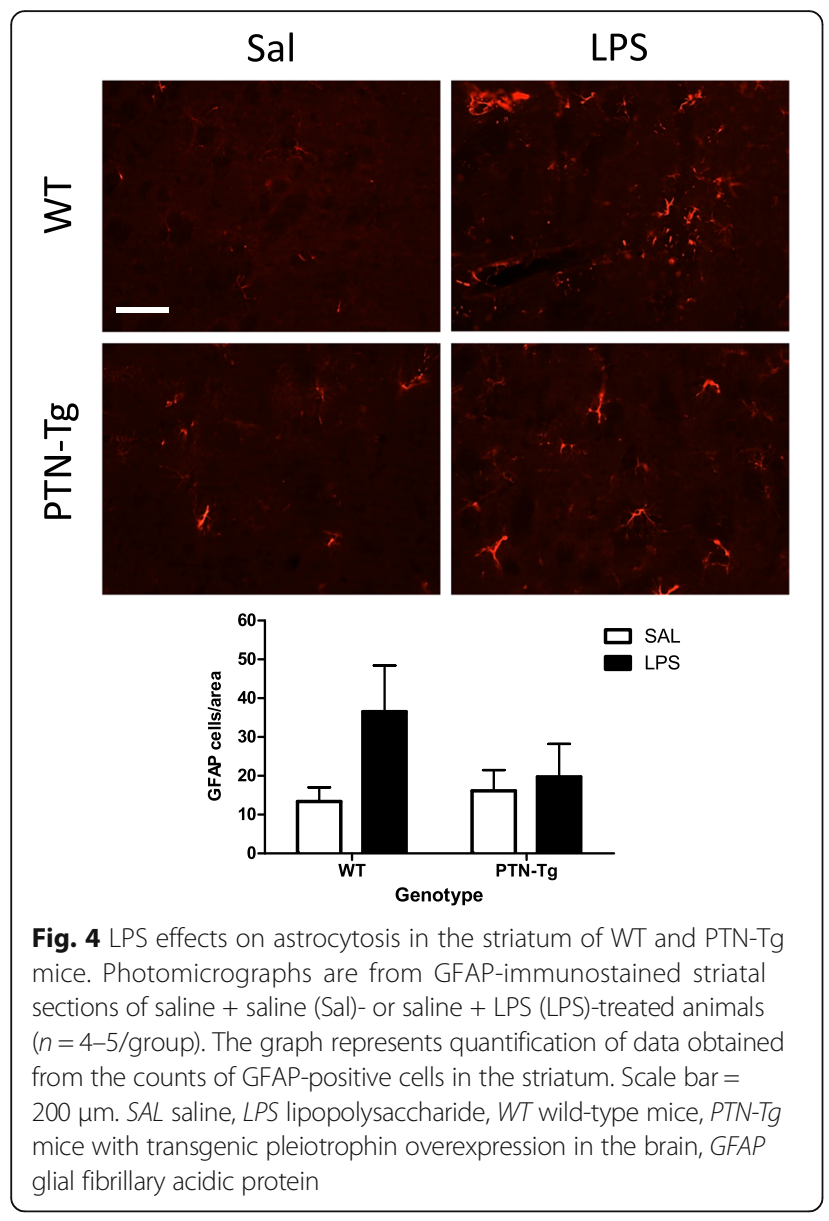

$[19,21]$. Interestingly, we recently found a highly significant (13-fold) increase in the number of GFAP+ astrocytes in amphetamine-treated PTN-Tg mice suggesting an enhanced neuroinflammatory response induced by amphetamine in the presence of higher levels of PTN [19]. Taking together, these data suggest that PTN differentially regulates the astrocytic response depending on the inflammatory stimulus.

Although upregulation of GFAP in astrocytes is considered an indicator of reactive astrogliosis and neuroinflammation, the regulation by PTN of two key elements of neuroinflammation, microglia activation, and cytokines release had not been tested before. In WT mice, LPS tended to increase the perimeter of Iba1+ cells and induced a significant increase in these cells area in the $\mathrm{PFC}$, indicating the presence of hypertrophic microglia. In addition, LPS induced significant increases in the levels of TNF- $\alpha$, IL- 6 , and MCP-1 in WT mice confirming LPS-induced neuroinflammation in normal mice. Interestingly, we found that PTN overexpression enhances microglial cell morphological changes to an activated-form in the PFC of LPS-treated PTN-Tg mice compared to that of WT mice. This was accompanied by more pronounced LPS-induced increases of TNF- $\alpha$,
IL-6, and MCP-1 in PTN-Tg mice compared to WT animals, indicating an important role of PTN on microglial activation and neuroinflammation. Interestingly, the TLR4 antagonist TAK-242 did not have any effect on LPS-induced microglia morphological changes in either genotype but efficiently blocked LPS-induced increases of TNF- $\alpha$, IL- 6 , and MCP- 1 in both genotypes. This is the first study showing that PTN overexpression in vivo significantly potentiates LPS-induced microglial activation and neuroinflammation. It is important to note that we did not find differences between genotypes in salinetreated animals suggesting that PTN is an important cytokine in the promotion of the effects triggered by the inflammatory stimulus but does not trigger proinflammatory cascades itself. Accordingly, our studies in vitro in murine BV2 microglia cells demonstrate that incubation with PTN alone does not cause significant effects on microglia. However, PTN significantly enhanced LPSinduced NO release in BV2 cells. Taking together, these studies may be of relevance since PTN is found highly upregulated in different brain disorders characterized by neuroinflammation such as Parkinson's disease [16], Alzheimer's disease [40], addictive disorders [14, 41, 42], tumors [43], and ischemia [44]. However, we must be cautious because the mechanisms involved in the shortterm inflammatory response induced by LPS might differ substantially from those involved in the long-term inflammation associated with the brain disorders mentioned above. In the present work, we have demonstrated for the first time that PTN potentiates microglial activation and neuroinflammation after an acute exposure to the inflammatory stimulus. Additional studies are needed to describe its role in chronic conditions of neuroinflammation.

Inflammation is a host defense mechanism mounted to reduce injury. However, prolonged or uncontrolled inflammation can lead to tissue damage and destruction. On the other hand, recent studies have shown that activated microglia contributes to the maintenance of tissue homeostasis and protection of the CNS under various pathological conditions [45]. Whether microglial activation primed by elevated levels of PTN contributes to the deleterious or homeostatic effects of microglia needs to be clarified. However, it has to be noted that one of the mechanisms that microglia adopts to downregulate inflammation is the production of anti-inflammatory mediators such as IL-4 and IL-10, which suppress the function of proinflammatory cytokines [46]. Here, we demonstrate that the content of IL-10 and IL- 4 is not affected by increased PTN levels in the PFC of PTN-Tg mice, whereas the proinflammatory cytokines TNF- $\alpha$, IL-6, and MCP-1 are highly upregulated compared to WT mice treated with LPS. Thus, our data suggest that high PTN levels lead activated microglia to promotion of neuroinflammation rather than suppression. 
A
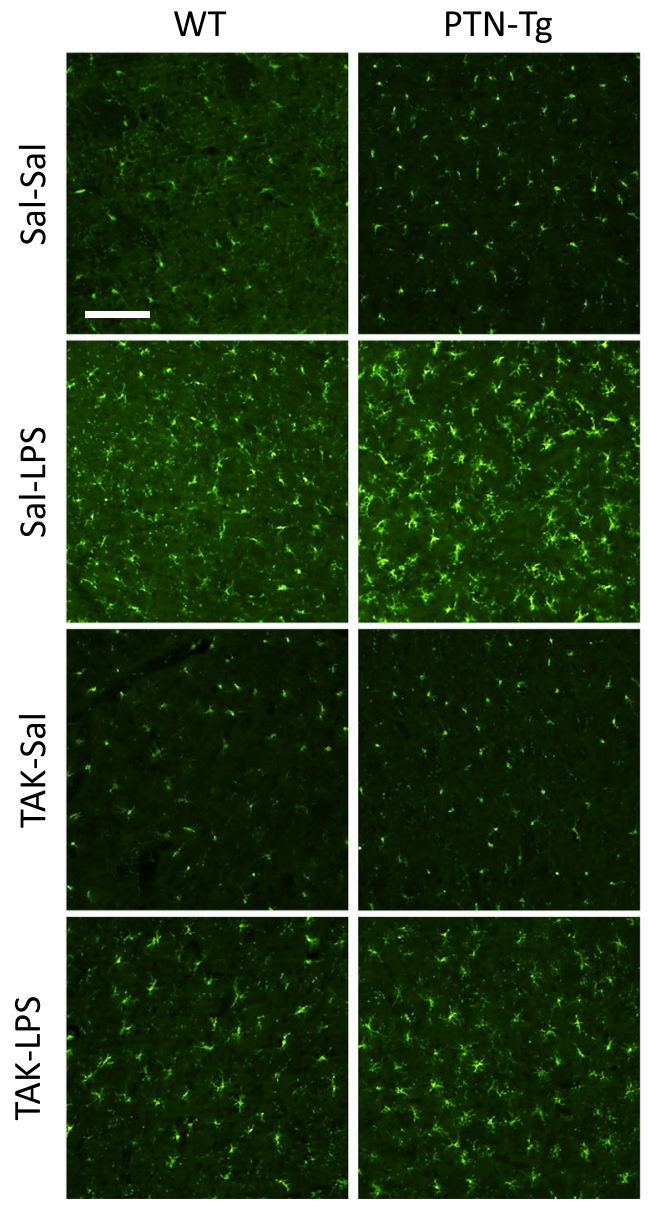
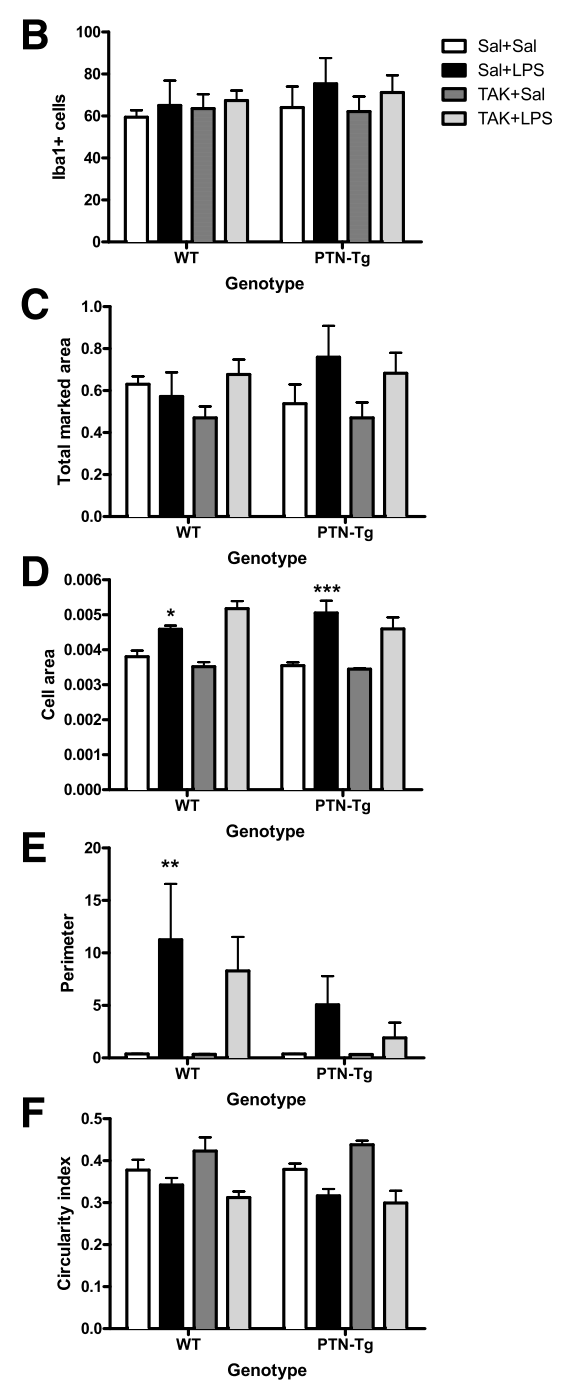

Fig. 5 Effects of TAK-242 and LPS on microglia activation in the striatum of PTN-Tg mice. Photomicrographs are from Iba-1-immunostained striatal sections of saline (Sal)- or TAK-242 (TAK)-pretreated and saline (Sal)- or LPS-treated animals ( $n=4-5 /$ group) (a). Graphs represent quantification of data (mean \pm SEM) obtained from the counts of Iba-1-positive cells $(\mathbf{b})$, total marked area (c), cell area (d), soma perimeter (e), and circularity index (f) in the striatum. ${ }^{*} P<0.05,{ }^{* *} P<0.01,{ }^{* *} P<0.001$ vs. Sal + Sal within the same genotype. Scale bar $=100 \mu \mathrm{m}$. SAL saline, LPS lipopolysaccharide, TAK TAK-242, WT wild-type mice, PTN-Tg mice with transgenic pleiotrophin overexpression in the brain, Ibal ionized calcium-binding adaptor molecule 1

The mechanism of action of PTN supports our findings. PTN binds receptor protein tyrosine phosphatase (RPTP) $\beta / \zeta$ (a.k.a. PTPRZ1) [47] and inactivates its phosphatase activity. Inhibition of the phosphatase activity of RPTP $\beta / \zeta$ by PTN binding regulates the tyrosine phosphorylation of substrates of $\mathrm{RPTP} \beta / \zeta$ that are known regulators of neuroinflammation such as Fyn kinase [48]. After stimulation with LPS, Fyn is activated in microglia by increased phosphorylation in Y416 [48]. Activated Fyn phosphorylates PKC $\delta$ at Y311, contributing to an increase in its kinase activity. The Fyn-PKC $\delta$-signaling axis further activates the LPS-induced MAP kinase phosphorylation and activation of the NFKB pathway, implying that Fyn is a major upstream regulator of proinflammatory signaling [48]. These signaling events are observed as well in animal models of PD [48]. Previously, we demonstrated that PTN is a key modulator of tyrosine phosphorylation of Fyn [49], suggesting that PTN could be a major upstream regulator of microglial neuroinflammatory processes in all those neurological disorders in which significantly increased levels of PTN have been detected by triggering inflammogen-induced increase in Fyn kinase activity. 


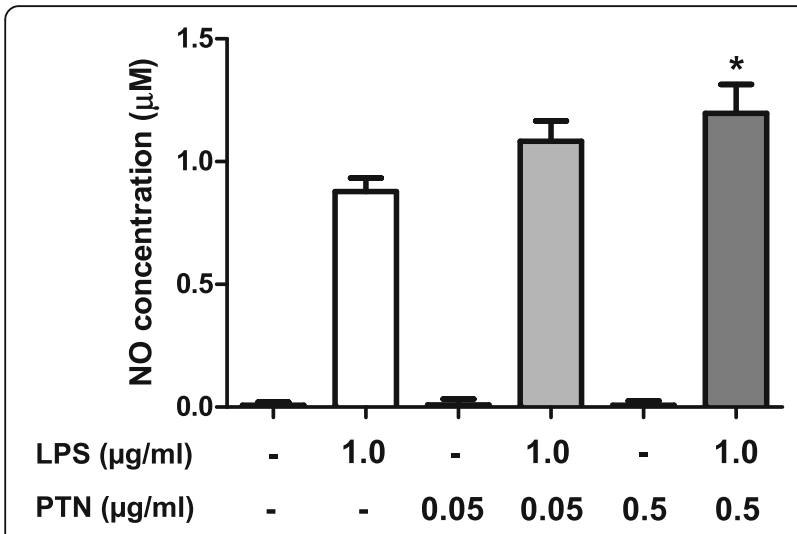

Fig. 6 Effects of PTN on LPS-induced NO production in BV2 microglial cells. Cells were treated with the indicated concentrations of PTN $(0.05$ or $0.5 \mu \mathrm{g} / \mathrm{ml})$ and/or with LPS $(1.0 \mu \mathrm{g} / \mathrm{ml})$ for $24 \mathrm{~h}$. The data of the levels of $\mathrm{NO}$ in the media are expressed as the mean \pm SEM. ${ }^{*} P<0.05$ vs. cells treated with LPS. NO nitric oxide, LPS lipopolysaccharide, PTN pleiotrophin

\section{Conclusions}

Our findings identify for the first time that PTN is a novel and potent regulator of neuroinflammation. Pleiotrophin potentiates LPS-stimulated microglia activation. Our results indicate that the regulation of PTN signaling pathways may constitute new therapeutic opportunities particularly in those neurological disorders characterized by increased PTN cerebral levels and neuroinflammation.

\section{Abbreviations}

ANOVA: Analysis of variance; BBB: Blood-brain barrier; BCA: Bicinchoninic acid; CNS: Central nervous system; COX2: Cyclooxygenase-2 enzyme; GFAP: Glial fibrillary acidic protein; Iba1: lonized calcium-binding adaptor molecule 1 ; IL10: Interleukin 10; IL-13: Interleukin 13; IL-4: Interleukin 4; IL-6: Interleukin 6; iNOS: Inducible nitric oxide synthase; LPS: Lipopolysaccharide; MAP kinase: Mitogen-activated protein kinase; MCP-1: Monocyte chemoattractant protein-1; NFkB: Nuclear factor-kB; NO: Nitric oxide; PD: Parkinson's disease; PFC: Prefrontal cortex; PKC $\delta$ : Protein kinase C delta type; PTN-/-: PTN genetically deficient mice; PTN: Pleiotrophin; PTN-Tg: Mice with transgenic PTN overexpression in the brain; qRT-PCR: Quantitative real-time polymerase chain reaction; RPMI-1640: Roswell Park Memorial Institute 1640 medium; RPTP $\beta / \zeta$ (PTPRZ1): Receptor protein tyrosine phosphatase $\beta / \zeta$; SEM: Standard error of the mean; TLR4: Toll-like receptor 4; TLRs: Toll-like receptors; TNF-a: Tumor necrosis factor-a; WT: Wild type
\end{abstract}

\section{Acknowledgements}

This work has been supported by grants SAF2014-56671-R from the Ministerio de Economía y Competitividad of Spain, PNSD00112015 from the National Plan on Drug abuse, Ministerio de Sanidad of Spain, and USP-BS-APP03/2014 from the Universidad CEU San Pablo and Banco de Santander to GH. MVR and RFC are supported by fellowships from Fundación Universitaria San Pablo CEU.

\section{Funding}

SAF2014-56671-R (Ministerio de Economía y Competitividad of Spain), PNSD00112015 (National Plan on Drug abuse, Ministerio de Sanidad of Spain), and USP-BS-APP03/2014 (Universidad CEU San Pablo and Banco de Santander).

\section{Availability of data and materials}

The datasets used and/or analyzed during the current study are available from the corresponding author on reasonable request.

\section{Authors' contributions}

RFC, MVR, EG, and JP performed the experiments. CPG, MPR, and GH analyzed and interpreted the results. MFA and MU contributed with the development of the transgenic mouse model and data interpretation. $\mathrm{GH}$ wrote the manuscript. All authors read and approved the final manuscript.

\section{Competing interests}

The authors declare that they have no competing interests.

\section{Consent for publication}

Not applicable

\section{Ethics approval and consent to participate}

All the animals used in this study were maintained in accordance with the European Union Laboratory Animal Care Rules (86/609/ECC directive) and the protocols were approved by the Animal Research Committee of USP-CEU.

\section{Author details}

'Pharmacology Lab, Department of Pharmaceutical and Health Sciences, Facultad de Farmacia, Universidad CEU San Pablo, Urb. Montepríncipe, 28668 Boadilla del Monte, Madrid, Spain. ${ }^{2}$ Biochemistry and Molecular Biology lab, Department of Chemistry and Biochemistry, Facultad de Farmacia, Universidad CEU San Pablo, Madrid, Spain. ${ }^{3}$ BRAINco Biopharma, S.L., Bizkaia Technology Park, Vizcaya, Spain.

Received: 8 December 2016 Accepted: 24 February 2017

Published online: 04 March 2017

\section{References}

1. Barbierato M, Facci L, Argentini C, Marinelli C, Skaper SD, Giusti P. Astrocytemicroglia cooperation in the expression of a pro-inflammatory phenotype. CNS Neurol Disord Drug Targets. 2013;12:608-18.

2. Hanke ML, Kielian T. Toll-like receptors in health and disease in the brain: mechanisms and therapeutic potential. Clin Sci (Lond). 2011;121:367-87.

3. O'Neill LA. Therapeutic targeting of Toll-like receptors for inflammatory and infectious diseases. Curr Opin Pharmacol. 2003:3:396-403.

4. Akira S, Takeda K. Functions of toll-like receptors: lessons from KO mice. C R Biol. 2004;327:581-9.

5. Amor S, Peferoen LA, Vogel DY, Breur M, van der Valk P, Baker D, van Noort JM. Inflammation in neurodegenerative diseases-an update. Immunology. 2014;142:151-66.

6. Herradon G, Perez-Garcia C. Targeting midkine and pleiotrophin signalling pathways in addiction and neurodegenerative disorders: recent progress and perspectives. Br J Pharmacol. 2014;171:837-48.

7. Najjar S, Pearlman DM, Alper K, Najjar A, Devinsky O. Neuroinflammation and psychiatric illness. J Neuroinflammation. 2013;10:43.

8. Silva AP, Martins T, Baptista S, Goncalves J, Agasse F, Malva JO. Brain injury associated with widely abused amphetamines: neuroinflammation, neurogenesis and blood-brain barrier. Curr Drug Abuse Rev. 2003;3:239-54.

9. Clark KH, Wiley CA, Bradberry CW. Psychostimulant abuse and neuroinflammation: emerging evidence of their interconnection. Neurotox Res. 2013;23:174-88.

10. Moratalla R, Khairnar A, Simola N, Granado N, Garcia-Montes JR, Porceddu PF, Tizabi Y, Costa G, Morelli M. Amphetamine-related drugs neurotoxicity in humans and in experimental animals: main mechanisms. Prog Neurobiol. 2015; doi:10.1016/j.pneurobio.2015.09.011.

11. Curtin K, Fleckenstein AE, Robison RJ, Crookston MJ, Smith KR, Hanson GR. Methamphetamine/amphetamine abuse and risk of Parkinson's disease in Utah: a population-based assessment. Drug Alcohol Depend. 2015;146:30-8.

12. Hwang O. Role of oxidative stress in Parkinson's disease. Exp Neurobiol. 2013;22:11-7.

13. Nimmo AJ, Vink R. Recent patents in CNS drug discovery: the management of inflammation in the central nervous system. Recent Pat CNS Drug Discov. 2009;4:86-95.

14. Le Greves P. Pleiotrophin gene transcription in the rat nucleus accumbens is stimulated by an acute dose of amphetamine. Brain Res Bull. 2005;65: 529-32.

15. Alguacil LF, Herradon G. Midkine and pleiotrophin in the treatment of neurodegenerative diseases and drug addiction. Recent Pat CNS Drug Discov. 2015;10:28-33. 
16. Marchionini DM, Lehrmann E, Chu Y, He B, Sortwell CE, Becker KG, Freed WJ, Kordower JH, Collier TJ. Role of heparin binding growth factors in nigrostriatal dopamine system development and Parkinson's disease. Brain Res. 2007;1147:77-88.

17. Achour A, M'Bika JP, Baudouin F, Caruelle D, Courty J. Pleiotrophin induces expression of inflammatory cytokines in peripheral blood mononuclear cells. Biochimie. 2008;90:1791-5.

18. Silver K, Desormaux A, Freeman LC, Lillich JD. Expression of pleiotrophin, an important regulator of cell migration, is inhibited in intestinal epithelial cells by treatment with non-steroidal anti-inflammatory drugs. Growth Factors. 2012;30:258-66.

19. Vicente-Rodriguez M, Rojo-Gonzalez L, Gramage E, Fernandez-Calle R, Chen Y, Perez-Garcia C, Ferrer-Alcon M, Uribarri M, Bailey A, Herradon G. Pleiotrophin overexpression regulates amphetamine-induced reward and striatal dopaminergic denervation without changing the expression of dopamine D1 and D2 receptors: implications for neuroinflammation. Eur Neuropsychopharmacol. 2016;26:1794-805.

20. Gramage E, Rossi L, Granado N, Moratalla R, Herradon G. Genetic inactivation of pleiotrophin triggers amphetamine-induced cell loss in the substantia nigra and enhances amphetamine neurotoxicity in the striatum. Neuroscience. 2010;170:308-16

21. Gramage E, Putelli A, Polanco MJ, Gonzalez-Martin C, Ezquerra L, Alguacil LF, Perez-Pinera P, Deuel TF, Herradon G. The neurotrophic factor pleiotrophin modulates amphetamine-seeking behaviour and amphetamine-induced neurotoxic effects: evidence from pleiotrophin knockout mice. Addict Biol. 2010;15:403-12

22. Asari A, Kanemitsu T, Kurihara $\mathrm{H}$. Oral administration of high molecular weight hyaluronan $(900 \mathrm{kDa}$ ) controls immune system via Toll-like receptor 4 in the intestinal epithelium. J Biol Chem. 2010;285:24751-8.

23. Ferrer-Alcón M, Uribarri M, Díaz A, Del Olmo N, Valdizán EM, Gramage E, Martín M, Castro E, Pérez-García C, Mengod G, Maldonado R, Herradon G, Pazos A, Palacios JM. A new non-classical transgenic animal model of Depression Program No. 776.04/FF9 Neuroscience Meeting Planner. New Orleans: Society for Neuroscience; 2012. Online.

24. Vicente-Rodriguez M, Perez-Garcia C, Ferrer-Alcon M, Uribarri M, SanchezAlonso MG, Ramos MP, Herradon G. Pleiotrophin differentially regulates the rewarding and sedative effects of ethanol. J Neurochem. 2014;131:688-95.

25. Aigner L, Arber S, Kapfhammer JP, Laux T, Schneider C, Botteri F, Brenner HR, Caroni P. Overexpression of the neural growth-associated protein GAP43 induces nerve sprouting in the adult nervous system of transgenic mice. Cell. 1995:83:269-78.

26. Caroni P. Overexpression of growth-associated proteins in the neurons of adult transgenic mice. J Neurosci Methods. 1997;71:3-9.

27. Vicente-Rodriguez M, Perez-Garcia C, Haro M, Ramos MP, Herradon G. Genetic inactivation of midkine modulates behavioural responses to ethanol possibly by enhancing GABA(A) receptor sensitivity to $G A B A(A)$ acting drugs. Behav Brain Res. 2014;274:258-63.

28. Vicente-Rodriguez M, Herradon G, Ferrer-Alcon M, Uribarri M, Perez-Garcia C. Chronic cocaine use causes changes in the striatal proteome depending on the endogenous expression of pleiotrophin. Chem Res Toxicol. 2015;28: 1443-54.

29. Gramage E, Martin YB, Ramanah P, Perez-Garcia C, Herradon G. Midkine regulates amphetamine-induced astrocytosis in striatum but has no effects on amphetamine-induced striatal dopaminergic denervation and addictive effects: functional differences between pleiotrophin and midkine. Neuroscience. 2011;190:307-17.

30. Luo XL, Liu SY, Wang L, Zhang QY, Xu P, Pan LL, Hu JF. A tetramethoxychalcone from Chloranthus henryi suppresses lipopolysaccharide-induced inflammatory responses in BV2 microglia. Eur J Pharmacol. 2016;774:135-43.

31. Lastres-Becker I, Garcia-Yague AJ, Scannevin RH, Casarejos MJ, Kugler S, Rabano A, Cuadrado A. Repurposing the NRF2 activator dimethyl fumarate as therapy against synucleinopathy in Parkinson's disease. Antioxid Redox Signal. 2016;25:61-77.

32. Torika N, Asraf K, Danon A, Apte RN, Fleisher-Berkovich S. Telmisartan modulates glial activation: in vitro and in vivo studies. PLoS One. 2016;11: e0155823.

33. Frey D, Jung S, Brackmann F, Richter-Kraus M, Trollmann R. Hypoxia potentiates LPS-mediated cytotoxicity of BV2 microglial cells in vitro by synergistic effects on glial cytokine and nitric oxide system. Neuropediatrics. 2015:46:321-8
34. Hida $H$, Jung CG, Wu CZ, Kim HJ, Kodama Y, Masuda T, Nishino H. Pleiotrophin exhibits a trophic effect on survival of dopaminergic neurons in vitro. Eur J Neurosci. 2003;17:2127-34.

35. Himburg HA, Muramoto GG, Daher P, Meadows SK, Russell JL, Doan P, Chi JT, Salter AB, Lento WE, Reya T, Chao NJ, Chute JP. Pleiotrophin regulates the expansion and regeneration of hematopoietic stem cells. Nat Med. 2010;16:475-82.

36. Maragakis NJ, Rothstein JD. Mechanisms of disease: astrocytes in neurodegenerative disease. Nat Clin Pract Neurol. 2006;2:679-89.

37. Qin L, Liu Y, Wang T, Wei SJ, Block ML, Wilson B, Liu B, Hong JS. NADPH oxidase mediates lipopolysaccharide-induced neurotoxicity and proinflammatory gene expression in activated microglia. J Biol Chem. 2004; 279:1415-21.

38. Ezquerra L, Alguacil LF, Nguyen T, Deuel TF, Silos-Santiago I, Herradon G. Different pattern of pleiotrophin and midkine expression in neuropathic pain: correlation between changes in pleiotrophin gene expression and rat strain differences in neuropathic pain. Growth Factors. 2008;26:44-8.

39. Gramage E, Herradon G. Connecting Parkinson's disease and drug addiction: common players reveal unexpected disease connections and novel therapeutic approaches. Curr Pharm Des. 2011;17:449-61.

40. Wisniewski T, Lalowski M, Baumann M, Rauvala H, Raulo E, Nolo R, Frangione B. HB-GAM is a cytokine present in Alzheimer's and Down's syndrome lesions. Neuroreport. 1996;7:667-71.

41. Mailleux P, Preud'homme X, Albala N, Vanderwinden JM, Vanderhaeghen JJ. delta-9-Tetrahydrocannabinol regulates gene expression of the growth factor pleiotrophin in the forebrain. Neurosci Lett. 1994:175:25-7.

42. Herradon G, Ezquerra L, Gramage E, Alguacil LF. Targeting the pleiotrophin/ receptor protein tyrosine phosphatase beta/zeta signaling pathway to limit neurotoxicity induced by drug abuse. Mini Rev Med Chem. 2009;9:440-7.

43. Peria FM, Neder L, Marie SK, Rosemberg S, Oba-Shinjo SM, Colli BO, Gabbai AA, Malheiros SM, Zago MA, Panepucci RA, Moreira-Filho CA, Okamoto OK, Carlotti Jr CG. Pleiotrophin expression in astrocytic and oligodendroglial tumors and it's correlation with histological diagnosis, microvascular density, cellular proliferation and overall survival. J Neurooncol. 2007;84:255-61.

44. Miao J, Ding M, Zhang A, Xiao Z, Qi W, Luo N, Di W, Tao Y, Fang Y. Pleiotrophin promotes microglia proliferation and secretion of neurotrophic factors by activating extracellular signal-regulated kinase 1/2 pathway. Neurosci Res. 2012;74:269-76.

45. Chen Z, Trapp BD. Microglia and neuroprotection. J Neurochem. 2016;136 Suppl 1:10-7.

46. Colton CA. Heterogeneity of microglial activation in the innate immune response in the brain. J Neuroimmune Pharmacol. 2009;4:399-418.

47. Meng K, Rodriguez-Pena A, Dimitrov T, Chen W, Yamin M, Noda M, Deuel TF. Pleiotrophin signals increased tyrosine phosphorylation of beta betacatenin through inactivation of the intrinsic catalytic activity of the receptor-type protein tyrosine phosphatase beta/zeta. Proc Natl Acad Sci U S A. 2000;97:2603-8.

48. Panicker N, Saminathan $H$, Jin H, Neal M, Harischandra DS, Gordon R, Kanthasamy K, Lawana V, Sarkar S, Luo J, Anantharam V, Kanthasamy AG, Kanthasamy A. Fyn kinase regulates microglial neuroinflammatory responses in cell culture and animal models of Parkinson's disease. J Neurosci. 2015;35: 10058-77.

49. Pariser $H$, Ezquerra L, Herradon G, Perez-Pinera P, Deuel TF. Fyn is a downstream target of the pleiotrophin/receptor protein tyrosine phosphatase beta/zeta-signaling pathway: regulation of tyrosine phosphorylation of Fyn by pleiotrophin. Biochem Biophys Res Commun. 2005;332:664-9. 